\title{
Human Trichuriasis: Whipworm Genetics, Phylogeny, Transmission and Future Research Directions
}

\author{
Martha Betson $^{1} \cdot$ Martin Jensen Søe ${ }^{2} \cdot$ Peter Nejsum $^{3}$
}

Published online: 28 September 2015

(C) Springer International Publishing AG 2015

\begin{abstract}
Human trichuriasis is a neglected tropical disease which affects hundreds of millions of people worldwide and is particularly prevalent among children living in areas where sanitation is poor. This review examines the current knowledge on the taxonomy, genetics and phylogeography of human Trichuris and its relationship to whipworm parasites in other host species. The evidence for zoonotic transmission of Trichuris and the emergence of anthelmintic resistance is assessed. In addition, the implications of the recent publication of the genomes and transcriptomes of multiple Trichuris species are discussed. Finally, priorities for future research in Trichuris genetics are proposed.
\end{abstract}

Keywords Trichuris · Whipworm · Phylogenetics · Genomics · Ancient DNA · Soil-transmitted helminth · Zoonosis $\cdot$ Anthelmintic resistance

This article is part of the Topical Collection on Topics Exploring Loa-Loa, Onchocerciasis, Hookworm, Ascaris, Trichuris

Martha Betson

m.betson@surrey.ac.uk

Martin Jensen Søe

msoe@plen.ku.dk

Peter Nejsum

pn@sund.ku.dk

1 School of Veterinary Medicine, Faculty of Health and Medical Sciences, University of Surrey, Daphne Jackson Road, Guildford GU2 7AL, UK

2 Department of Plant and Environmental Sciences, Faculty of Science, University of Copenhagen, Thorvaldsensvej 40, 2-70, 3rd floor, 1871 Frederiksberg C, Denmark

3 Department of Veterinary Disease Biology, Faculty of Health Sciences, University of Copenhagen, Dyrlaegevej 100, 1870 Frederiksberg C, Denmark

\section{Introduction}

Human trichuriasis is a neglected tropical disease caused by the whipworm parasites of the genus Trichuris. Trichuris is a highly prevalent soil-transmitted helminth (STH) infection, with an estimated 465 million people infected worldwide [1•]. Infections are particularly prevalent among children living in communities where hygiene and sanitation are poor. Medium to heavy infections are generally associated with chronic morbidities including dysentery, abdominal pathology, stunting and wasting and reduced cognitive capacity, all of which can significantly perturb child development $[1 \bullet, 2]$. It has been estimated that trichuriasis contributes 0.64 million disability-adjusted life years (DALYs) to the global burden of disease [3]. Despite the prevalence and undoubted public health importance of human trichuriasis, the number of studies published on the genetics and phylogeography of the Trichuris parasite is limited, especially when compared to the giant roundworm Ascaris which has similar epidemiological characteristics [4]. One possible explanation for this paucity of publications could be the difficulty in obtaining parasite material. Adult Ascaris worms are large and can be readily collected from infected individuals through chemoexplusion. In contrast, adult Trichuris are much smaller and are embedded in the mucosa of the large intestine, which can make chemoexpulsion less efficient and expelled worms more difficult to spot [5].

\section{Taxonomy and Phylogenetics}

The genus Trichuris belongs to the class Enoplea and the order Trichinellida together with Trichinella spiralis. In the NCBI Taxonomy Browser, there are 16 designated species in the genus Trichuris that are found in different mammalian hosts. 
Trichuris suis in pigs is the most closely related species to Trichuris trichiura but whether Trichuris in dogs and rodents (Trichuris vulpis, Trichuris arvicolae, Trichuris muris) or ruminants (Trichuris ovis, Trichuris discolor, Trichuris skrjabini) diverged before or after Trichuris trichiura is not yet settled $[6,7,8 \bullet, 9 \bullet]$.

Whipworms in humans are traditionally designated Trichuris trichiura. However, recent publications have begun to address whether several species circulate in the human population and whether non-human primates (NHPs) share whipworms with humans. As the Trichuris genus has a wide geographical distribution and infects multiple host species, it is a likely candidate to contain cryptic species, meaning those that cannot be identified to species using traditional methods, including morphological examination [10].

Sampling of Trichuris from a range of wild and captive NHP host species, followed by sequencing of nuclear and mitochondrial markers, has allowed a more careful interrogation of the taxonomic relationship of Trichuris parasites in humans and NHP and the potential for cross-species transmission. Ravasi et al. sampled Trichuris from wild baboons on the Cape Peninsula in South Africa and compared internal transcribed spacer (ITS) sequences with those from human and pig Trichuris [11]. They found that the human Trichuris fell into two distinct clades (DG and CP-COB) both shared with Trichuris from baboons. Similar results were obtained by full mitochondrial DNA (mtDNA) genome analysis of worms from humans in China and Uganda and from baboons held in captivity in USA and Denmark [12]. Strikingly, the genetic distance between these worms was up to $20 \%$, and these studies suggest that Trichuris in humans comprises several species and that there is potential for parasite transmission between humans and baboons. Trichuris collected from captive baboons in Denmark and the USA were all found to fall in one phylogenetic group together with human Trichuris from Uganda based on sequence analysis of the conserved betatubulin gene, which appears to correspond to clade DG [13]. Analysis of Trichuris collected from humans and a range of NHP species living in and around Kibale National Park in Uganda revealed a similar separation of primate Trichuris into distinct clades to that described by Ravasi et al. [11]. Further substructuring of clade CP-GOB into two groups was suggested, one group comprising Trichuris from colobus and yellow-cheeked gibbon, and the other containing Trichuris from eight species of primate hosts including humans [14]. Sequencing of mitochondrial and nuclear markers of Trichuris collected from the endangered François' leaf-monkey indicated that this might represent a separate Trichuris species [15]. A very recent comprehensive study [8$\left[8^{\bullet}\right.$ has integrated new and published data into the phylogeny proposed by Callejon et al. [16]. The authors proposed that clade 2 contains two subclades, DG (baboon and human Trichuris) and MF (macaque Trichuris), and clade 1 has at least two subclades, CA
(Trichuris from range of primate species including humans) and SUIS (Trichuris from pigs and occasionally humans), with Trichuris from black-and-white colobus and gibbons falling into subclade CA or a separate grouping depending on the method used for phylogenetic tree building. Trichuris from black-and-white colobus has recently been described as a new Trichuris species based on morphology and named Trichuris colobae [17]. Our own analysis of the data used by Cavallero et al. but also including ITS sequences from François' leaf-monkey Trichuris identified the same clades and subclades and suggested that François' leaf-monkey Trichuris comprises its own subclade (see Fig. 1).

Whipworms in humans may therefore represent multiple Trichuris species, some of which are shared with NHPs or alternately comprise a species complex [8•,12]. More species or subspecies may be identified in the future as current studies have been hampered by small numbers of samples from humans in only a few localities and by different researchers using different genetic markers. Only recently, multiple Trichuris from humans from two geographically distinct areas, namely, China $(n=7)$ and Ecuador $(n=15)$, were genetically compared by sequencing the $r r n \mathrm{~L}$ gene of the mtDNA [19•]. A phylogeographic pattern was observed as worms from the same region clustered together, but whether they represent a subspecies remains unclear. There is an urgent need to sample additional worms from humans and NHPs from locations worldwide and apply multiple genetic markers to further explore the taxonomic relationship between worms. Previous studies have mainly relied on sequence analysis of the ITS regions $[8 \bullet, 11,14,20,21 \bullet]$, and as this region contains multiple repeats, the alignment of sequences even between closely related worms will include a number of gaps. This makes inference of the phylogenetic relationship between worms problematic, and it is therefore highly recommended to supplement such analysis with other genetic markers such as mitochondrial DNA genes [16].

\section{Zoonotic Transmission}

Although it is generally considered that most cases of human trichuriasis are attributable to the species Trichuris trichiura, there is some evidence of human infection with other Trichuris species and of zoonotic transmission of Trichuris parasites. Based on identification of its characteristically large eggs in human faecal samples, it has been suggested that Trichuris vulpis, which generally infects dogs and wolves, can be transmitted to humans [22]. However, there are natural variations in the size of Trichuris trichiura eggs, and females may produce large eggs which are similar in size to Trichuris vulpis eggs [22, 23], so conclusions based on egg size alone should be treated with caution. Using molecular markers, $11 \%$ of Trichuris-positive children in Thailand were shown 
Fig. 1 Phylogenetic tree based on sequences of the entire ITS region (ITS1-5.8S-ITS2) of

Trichuris from humans, nonhuman primates and other mammalian hosts [8•] and including ITS sequences from Trichuris from Francois' leafmonkey [14]. Sequences were aligned using MUSCLE, and a maximum likelihood tree was constructed in MEGA v6 [18], using the Tamura-Nei model with gamma distributed rates and including invariant sites. Ascaris lumbricoides was used as an outgroup and branch support is provided by bootstrapping ( 1000 replicates). Host species which have been associated with each subclade, either in this analysis or in published studies discussed in the main text, are indicated in brackets. NHP indicates a range of species including baboon, grivet, red-tailed monkey, L'Hoest's monkey, blue monkey, eastern red colobus, grey-cheeked mangabey and chimpanzee. Clades and subclades are labelled as in [8•]. Triangles indicate multiple sequences and the width of the triangle is proportional to the number of sequences. A new subclade (SUBCLADE-TF for Trachypithecus francoisi or François' leaf monkey) is proposed. A tree constructed using the neighbour joining method showed a similar topology, although in this case the proposed SUBCLADE-TF fell clearly within clade 2

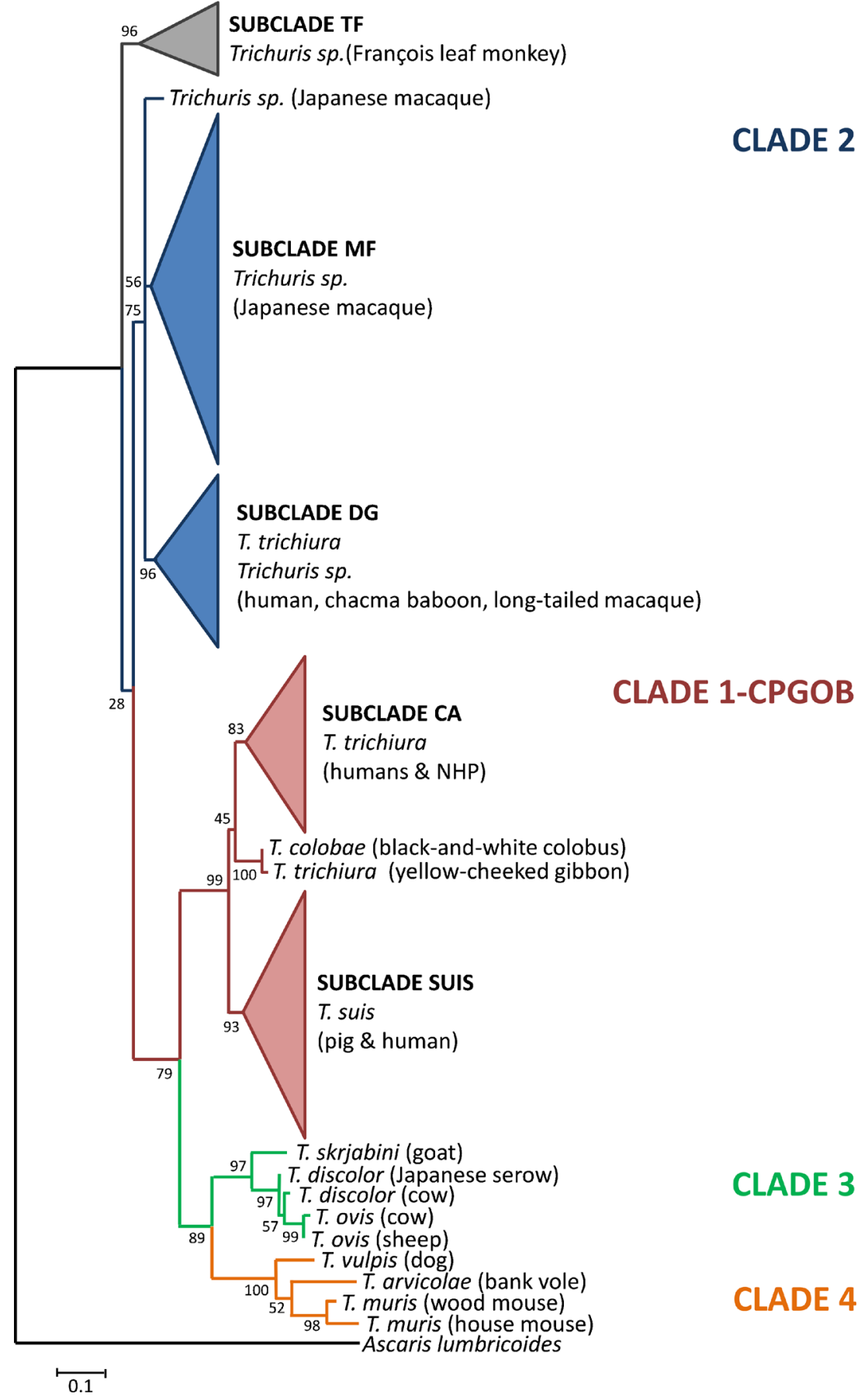

to have Trichuris vulpis eggs in their faeces, highlighting the possible zoonotic potential of this parasite in this region [24].

It is anticipated that Trichuris suis, which naturally infects pigs and can cause substantial economic losses in pig production, is a more obvious candidate for zoonotic transmission than Trichuris vulpis, as it is more closely related to Trichuris trichiura (see Fig. 1). However, morphological examination alone does not allow eggs, larvae and adult worms to be distinguished between Trichuris trichiura and Trichuris suis (reviewed in [25]). This means that potential cases of cross- transmission between host species cannot be identified using standard parasitological methods. Recently, there have been some efforts to apply molecular approaches to investigate the zoonotic potential of Trichuris suis. Nissen et al. (2012) demonstrated that 3 of 29 worms from humans living sympatrically with pigs in Uganda were in fact Trichuris suis [21•]. They used sequencing and PCR-RFLP analysis of the ITS-2 region, supplemented by sequencing of the beta-tubulin gene. In contrast, analysis of nuclear and mitochondrial markers in Trichuris sampled from pigs and humans in rural Ecuador 
did not find any evidence of zoonotic transmission of Trichuris suis in this setting [19•]. However, two pig Trichuris demonstrated a "heterozygous-type" PCR-RFLP pattern, suggesting that genetic exchange had taken place between Trichuris trichiura and Trichuris suis. Sequencing of the ITS region and the $r r n \mathrm{~L}$ gene of Trichuris derived from pigs and humans in China did not provide any evidence of crosstransmission between species; however, the number of samples $(n=16)$ examined was very limited $[9 \cdot, 26]$. Further sympatric sampling of Trichuris from humans and dogs or pigs in different geographical locations combined with molecular analysis is warranted and required to determine the extent and importance of transmission of Trichuris between domestic animals and humans.

Trichuris infections have been found in a range of NHP species living in natural habitats including colobus monkeys, macaques, baboons and chimpanzees [27-29]. Based on molecular studies as outlined above, some Trichuris species seem to be specific to particular NHPs whereas others most likely have the potential to circulate between humans and NHPs as they are genetically identical. This is particularly important when humans and NHPs are living in close proximity, as is becoming increasingly common with human encroachment into pristine habitats and NHPs accessing gardens and farms in search of food, and has significant implications for both human health and wildlife conservation.

\section{Anthelmintic Resistance}

Regular mass distribution of benzimidazole anthelminthic drugs, i.e. single-dose albendazole or mebendazole, to school-aged children is the mainstay of control of human trichuriasis [30], and there is currently a scale-up of deworming programmes to reach the WHO target of providing preventive chemotherapy to $75 \%$ of all preschool- and school-age children at risk by 2020 [4, 31]. However, when used as single doses, albendazole and mebendazole are not very effective for treatment of Trichuris, with cure rates in the order of 30-70\%. Concerns about the development of drug resistance have also been raised [2, 5, 32, 33]. The frequent use of anthelmintics for treatment of nematodes of veterinary importance, such as gastrointestinal nematodes of ruminants and horses, has led to the widespread development of anthelmintic resistance [34-37].

Resistance to benzimidazole drugs is commonly associated with a single SNP in the parasite beta-tubulin gene leading to substitution of phenylalanine by tyrosine at codon 200 [38]. Non-synonymous SNPs at codons 167 and 198 are also occasionally associated with resistance $[39,40]$. Initial work investigating sequence variation in the beta-tubulin gene of 72 Trichuris trichiura isolates from seven countries did not identify any mutations indicative of benzimidazole resistance [41].
It was suggested that anthelmintic resistance was likely to arise less rapidly in Trichuris trichiura than in trichostrongyles of farmed animals due its lower genetic diversity and smaller effective population size. More recently, pyrosequencing assays have been developed to detect nonsynonymous SNPs at codons 167, 198 and 200 in Trichuris trichiura and other soil-transmitted helminths [42, 43]. Among Trichuris sampled from Kenyan children who were believed not to have received benzimidazole treatment, $2.6 \%$ were homozygous for the resistance mutation at codon 200 suggesting that these SNPs are naturally occurring in the whipworm populations. In addition, five out of eight Trichuris trichiura egg pools collected from children treated with a single dose of albendazole contained only the codon 200 mutation associated with resistance [42]. In Trichuris trichiura samples collected before and after treatment with albendazole in Kenya and Haiti, there was a significant increase of the homozygous resistance genotype at codon 200 of betatubulin following treatment, and this was associated with poor egg reduction rates in both locations [44•]. Polymorphisms were also detected at codons 198 and 167 in Trichuris from Panama. In contrast, Hansen et al. did not detect any SNPs at beta-tubulin codons 167, 198 and 200 in Trichuris from humans in Uganda, domestic animals, wildlife and captive baboons [13, 45]. However, $41 \%$ of the human Trichuris samples were collected by chemoexpulsion using mebendazole, which may partly explain the absence of resistance SNPs. It is likely that when using DNA extracted from expelled worms, the resistance markers are less frequently observed as only worms without the resistance marker will be expelled. In contrast, screening of eggs from faeces will provide insights into the beta-tubulin genotypes of resistant and non-resistant female worms which produced the eggs.

In conclusion, although the data to date on whipworms do demonstrate the presence of SNPs in the beta-tubulin gene which are associated with anthelmintic resistance in other nematodes, more studies are required to definitively demonstrate that benzimidazole treatment is exerting selective pressure to increase the frequency of these mutations and that these SNPs are responsible for reduced efficacy of albendazole and mebendazole in treatment of human trichiuriasis. Other, not mutually exclusive, explanations for poor performance of anthelmintics could include the location of adult Trichuris trichiura in the large intestine as the drug has to pass through most of the gastrointestinal system before it reaches the parasite. Another factor influencing poor treatment outcomes may be the fact that that the anterior part of the Trichuris trichiura worm is embedded in the intestinal mucosa. Because of this, although worms may be paralysed by the anthelmintic, they are less likely to be expelled as they are attached to the host and can recover once the treatment has passed through the system. Also, while it is thought that the drug enters the worms through passive diffusion [46], 
Trichuris may be able to excrete the drug through Pglycoprotein-mediated transport [47].

\section{Ancient DNA}

Paleoparasitological studies have provided evidence for human infection with Trichuris for thousands of years even in places where infections are no longer prevalent, such as Europe and North America. This is based on the recovery of Trichuris eggs in archeological contexts such as human intestinal content, coprolites, latrine or environmental samples [48-52]. Traditionally, paleoparasitological studies rely on morphological assessments of parasite eggs, which limit identifications to genus level as discussed briefly above, in the section "Zoonotic Transmission." The major hurdle with ancient samples is degradation of DNA into small fragments that are not amplifiable using traditional PCR. Recently, typing of ancient DNA from parasite eggs has become feasible through advances in DNA extraction methods and the amplification and sequencing of short species-specific amplicons [53, 54]. In this regard, targeting small overlapping sequences of Trichuris 18S SSUrRNA has made it possible to reconstruct longer sequences from excavations in Denmark (1030 AD) and Korea (1755 AD) homologous to Trichuris trichiura $\left[54,55^{\bullet}\right]$. Continuing technological advances should make it possible to gain more detailed molecular insights into ancient parasites shedding light on prehistorical distributions and dispersal patterns.

\section{Genomics and Transcriptomics}

With the advent of advanced and high-throughput genomic and transcriptomic tools, we are now moving into a new era where a fundamentally novel approach can be used to obtain unprecedented insight into the molecular biology of parasites [56]. The genomes of Trichuris trichiura, Trichuris muris, and Trichuris suis have recently been published and represent a

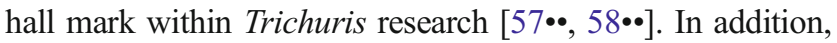
for Trichuris suis and Trichuris muris, the genomes were supported by transcriptome analysis thereby providing deep insights into the molecules and genes these parasites express as they invade, live in and interact with their hosts.

The genome of Trichuris trichiura is about 75.2 MB in size and encodes 9650 genes and is therefore smaller in size and gene number than Trichuris suis (male 83.6 MB, 14,781 genes, and female 87.2 MB, 14,470 genes) and Trichuris muris (85.0 MB and 11,004 genes), with the majority of gene families shared between Trichuris muris and Trichuris trichiura [57••]. The karyotype of Trichuris muris comprises two pairs of autosomes and one pair of $\mathrm{X}$ and $\mathrm{Y}$ sex chromosomes $(2 n=6)$, and it appears that this also is the case for
Trichuris trichiura. The genomes encode a high number of genes likely to be involved in parasite-host interactions and in immuno-regulation (or immuno-modulation) of the infected host. For example, 618 excretory-secretory proteins were predicted for Trichuris suis, and while secretory proteins make up about $4 \%$ of the gene set, they represent about $10 \%$ of the transcribed genes [58••]. Also, the anterior end of the worms which is embedded in host mucosal tissue produces proteases and protease inhibitors which were overexpressed in both Trichuris muris and Trichuris suis suggesting a central role of these molecules in host-tissue degradation and in regulating protein functions associated with immunomodulation [57••, $58 \bullet \bullet$.

Genomic and transcriptomic data also offer possibilities to discover novel drugs by targeting molecules that are vital and unique to the parasite, which is referred to as genome-guided drug discovery [59]. For both Trichuris suis and Trichuris trichiura, hundreds of potential drug targets were identified, and in Foth et al., this list was further narrowed down to contain 29 top protein candidates all representing homologues to targets for existing approved drugs [57••]. The function of small non-coding RNAs in gene regulation was investigated by Jex et al. [58••], and due to their central role as gene regulators, these RNAs species may also serve as novel drug targets [60].

\section{Future Research Directions}

Novel insights into Trichuris biology are expected in the coming years due to lower assay cost and easier implementation of "-omic" based approaches, including genomic, transcriptomic, epigenomic and proteomic studies. Advances have opened the way for comparative studies of wholegenome sequences at a population level [61, 62], which promise to revolutionize evolutionary and phylogeographic studies of Trichuris. This will lead to identification of new subspecies and hybridization events between existing species and enable a much clearer and more detailed understanding of dispersal patterns.

The major obstacle to genetic research on Trichuris will likely continue to be the availability of worms as anthelmintic treatments are not very effective. However, whole-genome sequencing of Trichuris trichiura [57••] has already identified a number of alternate drug candidates, which could make worm sampling easier in the future. Nonetheless, it is likely that readily available faecal samples containing Trichuris eggs will become integral in large-scale comparative studies as the required input sample is decreasing rapidly. Whole-genome sequences and even transcriptomes have been generated from single cells $[63,64]$, which should allow for similar studies on embryonated eggs that contain hundreds of cells in the near future. A first step could be micromanipulation that has 
allowed researchers to select individual helminth eggs for subsequent PCR-based amplification [45, 65].

Despite the fact that there seems to be a pattern of infection with different Trichuris species infecting particular host species (Fig. 1), there are several issues that need to be further explored. Are humans infected with a single or multiple Trichuris spp. and, if so, can they be found in the same area and do they differ with respect to pathology, epidemiology and drug susceptibility? Is there natural Trichuris transmission between NHP and humans and, if so, to what extent? Are the different Trichuris species in primates host specific, i.e. can Trichuris in one clade infect hosts of the other clades? We have just started to unravel some of these questions, and there is a need for further studies applying multiple genetic marker makers to Trichuris collected from humans and NHPs from sympatric areas and worldwide locations. This will illuminate parasite transmission routes between these primates and allow implementation of appropriate control and prevention measures.

Unravelling the mechanisms underlying host-parasite interactions is another key future research area, which is currently being pursued on a broader scale by 'the 50 Helminth Genomes project' [66] and in the '959 Nematode Genomes' initiative (http://www.nematodes.org/nematodegenomes/ index.php). These genomes will provide researchers with deep and unprecedented insight into the molecular biology and evolution of helminth parasites. One poorly understood mechanism in Trichuris development is that of host stimulusinduced egg hatching during passage though the small intestine. This has been shown to be highly host-parasite specific as Trichuris suis eggs require different stimuli to Trichuris muris for hatching [67-69]. Other mechanisms relate to larval establishment and evasion of host-immune responses, which are better understood today due to the Trichuris suis and Trichuris muris genome and transcriptome data [57••, 58••]. Trichuris trichiura transcriptome data will undoubtedly also provide new insights but are not yet published.

Exosomes are small $(30-100 \mathrm{~nm})$ microvesicles used by complex organisms for cell-cell communication. Intriguingly, a few recent studies have shown that parasites also produce and excrete exosomes containing microRNA which can be internalized by host cells [70-72]. Interestingly, Buck et al. (2014) have shown that exosomes secreted by the gastrointestinal nematode Heligmosomoides polygyrus suppress the type 2 innate response in mice and activate specific immune genes in vitro [73]. It was also shown that Trichuris suis also secretes vesicles in the size of exosomes and that these contain RNA, although their specific function remains unknown [74]. Future studies should therefore further characterize the contents of these exosomes and explore their function in the hostparasite interplay, e.g. whether Trichuris use exosomes for manipulating the host's immune response in order to dampen inflammation and optimize its survival.
In paleoparasitology, genotyping of ancient Trichuris eggs is likely to be carried out with increasing frequency. As Trichuris spp. have a narrow host range, genotyping a given egg finding has been suggested as a good way to identify the host of origin [55•], which provides paleoparasitologists with a tool to address prehistoric interactions between hosts. Furthermore, genotyping Trichuris eggs will provide solid anchor points in phylogeographic studies of prehistoric dispersal patterns. In this regard, Trichuris trichiura dispersal into the Americas is still very much debated [75]. The conundrum is that Trichuris trichiura, like other STH infections, can most likely not be maintained in Arctic climates which the human migration across the Beringian Land Bridge into the Americas would have necessitated [76, 77].

Finally, molecular diagnostics at the point of care is likely to become available for human trichuriasis, possibly in conjunction with other STH infections. It is imperative that testing can be performed in developing world settings with limited infrastructure and at low cost. Initial studies have identified loop-mediated isothermal amplification (LAMP) [78], or variants thereof $[79,80]$, as suitable technologies. Detection of DNA from Strongyloides stercoralis [81] and Opisthorchis viverrini [82] extracted from human stool samples has been achieved using LAMP. At present, the major hurdle lies in sample preparation and its integration with DNA extraction and detection [83]. This has been successfully accomplished in one device for testing of gastrointestinal pathogens, but so far not for any STHs [18, 84]. Large-scale point-of-care testing of human stool samples for STHs with molecular-based methods also raises the possibility of addressing the zoonotic potential of Trichuris spp. and revealing to what extent and with which species humans are actually infected.

\section{Conclusion}

Over the past few years, research has shed new light on human trichuriasis and the genetics of the Trichuris parasite. Significant results include publication of the Trichuris trichiura genome, as well as the Trichuris suis and Trichuris muris genomes and transcriptomes. This has identified important potential mechanisms of host-parasite interaction and immunomodulation, and possible novel drug targets. Alleles in the beta-tubulin gene associated with resistance in nematodes of veterinary importance have only been identified in a handful of studies on human Trichuris trichuira isolates. Recent progress has been made in understanding the phylogeny of the Trichuris genus. There seem to be host-specific patterns in infection with particular Trichuris species or subspecies. However, there is evidence of zoonotic transmission, especially regarding Trichuris trichiura infections in NHPs and possibly also for Trichuris suis in pigs and Trichuris vulpis in dogs. Future research priorities should include multiple 
genetic marker analysis of Trichuris sampled from humans, NHPs, pigs and dogs in sympatric and diverse geographical locations to provide insights into parasite transmission within and between host species. This will also open up new possibilities for monitoring the emergence of anthelmintic resistance and will aid in the development of point-of-care molecular tests for sensitive diagnosis. Progress in these areas will greatly enhance control of this widespread parasitic disease.

Acknowledgments We are very grateful to Prof. Xing-Quan Zhu and Dr. Guo-Hua Liu for providing ITS sequences from Trichuris from François leaf monkey.

\section{Compliance with Ethics Guidelines}

Conflict of Interest Martha Betson, Martin Jensen Søe and Peter Nejsum declare that they have no conflict of interest.

Human and Animal Rights and Informed Consent This article does not contain any studies with human or animal subjects performed by any of the authors.

\section{References}

Papers of particular interest, published recently, have been highlighted as:

- Of importance

•- Of major importance

1. Pullan RL, Smith JL, Jasrasaria R, Brooker SJ. Global numbers of infection and disease burden of soil transmitted helminth infections in 2010. Parasit Vectors. 2014;7:37. Comprehensive summary of soil-transmitted helminth prevalence data for 6091 locations in 118 countries.

2. Bethony J, Brooker S, Albonico M, Geiger SM, Loukas A, Hotez PJ. Soil-transmitted helminth infections: Ascaris, Trichuris, and hookworm. Lancet. 2006;367:1521-32.

3. Murray CJ, Vos T, Lozano R, Naghavi M, Flaxman AD, Michaud C, et al. Disability-adjusted life years (DALYs) for 291 diseases and injuries in 21 regions, 1990-2010: a systematic analysis for the Global Burden of Disease Study 2010. Lancet. 2012;380:2197223.

4. World Health Organization. Accelerating work to overcome the global impact of neglected tropical diseases - a roadmap for implementation. Geneva; 2012.

5. Olsen A, Namwanje H, Nejsum P, Roepstorff A, Thamsborg SM. Albendazole and mebendazole have low efficacy against Trichuris trichiura in school-age children in Kabale District, Uganda. Trans R Soc Trop Med Hyg. 2009;103:443-6.

6. Callejón R, De Rojas M. Feliú, Balao F, Marrugal A, Henttonen H, et al. Phylogeography of Trichuris populations isolated from different Cricetidae rodents. Parasitology. 2012;139:1795-812.

7. Callejón R, Gutiérrez-Avilés L, Halajian A, Zurita A, de Rojas M, Cutillas C. Taxonomy and phylogeny of Trichuris globulosa Von Linstow, 1901 from camels - a review of Trichuris species parasitizing herbivorous. Infect Genet Evol. 2015;34:61-74.

8. Cavallero S, De Liberato C, Friedrich KG, Di Cave D, Masella V, D'Amelio S, et al. Genetic heterogeneity and phylogeny of Trichuris spp. from captive non-human primates based on ribosomal DNA sequence data. Infect. Genet. Evol. [Internet]. 2015; Available from: http://linkinghub.elsevier.com/retrieve/pii/ S1567134815002415. Most recent and comprehensive phylogenetic analysis of Trichuris from humans and NHPs based on ITS sequences, demonstrating that primate Trichuris falls into at least four distinct sub-clades.

9. Liu GH, Gasser RB, Su A, Nejsum P, Peng L, Lin RQ, et al. Clear genetic distinctiveness between human- and pig-derived Trichuris based on analyses of mitochondrial datasets. PLoS Negl Trop Dis. 2012;6:e1539. Complete mitochondrial genomes of $\boldsymbol{T}$. trichiura and $T$. suis show clear genetic distinctiveness and provide strong support for the hypothesis that Trichuris in humans and pigs represents separate species.

10. Nadler S, De Leon G. Integrating molecular and morphological approaches for characterizing parasite cryptic species: implications for parasitology. Parasitology. 2011;138:1688-709.

11. Ravasi DF, O'Riain MJ, Davids F, Illing N. Phylogenetic evidence that two distinct Trichuris genotypes infect both humans and nonhuman primates. PLoS One. 2012;7:e44187.

12. Hawash MB, Anderson L, Gasser RB, Stensvold C, Nejsum P. Mitochondrial genome analyses suggest multiple Trichuris species in humans, baboons, and pigs from different geographical regions. PLoS Negl. Trop. Dis. 2015;9:e4059.

13. Hansen TVA, Nejsum P, Olsen A, Thamsborg SM. Genetic variation in codons 167, 198 and 200 of the beta-tubulin gene in whipworms (Trichuris spp.) from a range of domestic animals and wildlife. Vet Parasitol. 2013;193:141-9.

14. Ghai RR, Simons ND, Chapman CA, Omeja PA, Davies TJ, Ting $\mathrm{N}$, et al. Hidden population structure and cross-species transmission of whipworms (Trichuris sp.) in humans and non-human primates in Uganda. PLoS Negl Trop Dis. 2014;8:e3256.

15. Liu GH, Gasser RB, Nejsum P, Wang Y, Chen Q, Song HQ, et al. Mitochondrial and nuclear ribosomal DNA evidence supports the existence of a new Trichuris species in the endangered François' leaf-monkey. PLoS One. 2013;8:e66249.

16. Callejón R, Nadler S, De Rojas M, Zurita A, Petrášová J, Cutillas C. Molecular characterization and phylogeny of whipworm nematodes inferred from DNA sequences of cox $1 \mathrm{mtDNA}$ and $18 \mathrm{~S}$ rDNA. Parasitol Res. 2013;112:3933-49.

17. Cutillas C, De Rojas M, Zurita A, Oliveros R, Callejón R. Trichuris colobae n. sp. (Nematoda: Trichuridae), a new species of Trichuris from Colobus guereza kikuyensis. Parasitol Res. 2014;113:272532.

18. Tamura K, Stecher G, Peterson D, Filipski A, Kumar S. MEGA6: Molecular Evolutionary Genetics Analysis version 6.0. Mol Biol Evol. 2013;30:2725-9.

19. Meekums H, Hawash MB, Sparks AM, Oviedo Y, Sandoval C, Chico ME, et al. A genetic analysis of Trichuris trichiura and Trichuris suis from Ecuador. Parasit Vectors. 2015;8:168. First phylogenetic comparison of human Trichuris from Asia and South America.

20. Cutillas C, Callejón R, de Rojas M, Tewes B, Ubeda JM, Ariza C, et al. Trichuris suis and Trichuris trichiura are different nematode species. Acta Trop. 2009;111:299-307.

21. Nissen S, Al-Jubury A, Hansen TVA, Olsen A, Christensen H, Thamsborg SM, et al. Genetic analysis of Trichuris suis and Trichuris trichiura recovered from humans and pigs in a sympatric setting in Uganda. Vet Parasitol. 2012;188:68-77. First molecular comparison of Trichuris obtained from humans and pigs living in close proximity. Evidence for zoonotic transmission of $T$. suis is presented.

22. Traversa D. Are we paying too much attention to cardio-pulmonary nematodes and neglecting old-fashioned worms like Trichuris vulpis? Parasit Vectors. 2011;4:32.

23. Yoshikawa H, Yamada M, Matsumoto Y, Yoshida Y. Variations in egg size of Trichuris trichiura. Parasitol Res. 1989;75:649-54. 
24. Areekul P, Putaporntip C, Pattanawong U, Sitthicharoenchai P, Jongwutiwes S. Trichuris vulpis and T. trichiura infections among schoolchildren of a rural community in northwestern Thailand: the possible role of dogs in disease transmission. Asian Biomed. 2010;4:49-60.

25. Nejsum P, Betson M, Bendall RP, Thamsborg SM, Stothard JR. Assessing the zoonotic potential of Ascaris suum and Trichuris suis: looking to the future from an analysis of the past. J Helminthol. 2012;86:148-55.

26. Liu GH, Zhou W, Nisbet AJ, Xu MJ, Zhou DH, Zhao GH, et al. Characterization of Trichuris trichiura from humans and T. suis from pigs in China using internal transcribed spacers of nuclear ribosomal DNA. J Helminthol. 2014;88:64-8.

27. Arizono N, Yamada M, Tegoshi T, Onishi K. Molecular identification of Oesophagostomum and Trichuris eggs isolated from wild Japanese macaques. Korean J Parasitol. 2012;50:253-7.

28. Gillespie TR, Lonsdorf EV, Canfield EP, Meyer DJ, Nadler Y, Raphael J, et al. Demographic and ecological effects on patterns of parasitism in eastern chimpanzees (Pan troglodytes schweinfurthii) in Gombe National Park, Tanzania. Am J Phys Anthropol. 2010;143:534-44.

29. Kooriyama T, Hasegawa H, Shimozuru M, Tsubota T, Nishida T, Iwaki T. Parasitology of five primates in Mahale Mountains National Park Tanzania. Primates. 2012;53:365-75.

30. World Health Organization. Helminth control in school-age children. A guide for managers of control programmes. Geneva; 2011.

31. Uniting to Combat Neglected Tropical Diseases. Delivering on promises and driving progress. 2014.

32. Geerts S, Gryseels B. Anthelmintic resistance in human helminths: a review. Tropical Med Int Health. 2001;6:915-21.

33. Keiser J, Utzinger J. Efficacy of current drugs against soiltransmitted helminth infections: systematic review and meta-analysis. JAMA. 2008;299:1937-48.

34. Kaplan RM, Vidyashankar AN. An inconvenient truth: global worming and anthelmintic resistance. Vet Parasitol. 2012;186:708.

35. Sargison ND. Pharmaceutical treatments of gastrointestinal nematode infections of sheep-future of anthelmintic drugs. Vet Parasitol. 2012;189:79-84.

36. Sutherland IA, Leathwick DM. Anthelmintic resistance in nematode parasites of cattle: a global issue? Trends Parasitol. 2011;27: 176-81.

37. Matthews J. Anthelmintic resistance in equine nematodes. Int $\mathbf{J}$ Parasitol Drugs Drug Resist. 2014;4:310-5.

38. Kwa MSG, Veenstra JG, Roos MH. Benzimidazole resistance in Haemonchus contortus is correlated with a conserved mutation at amino acid 200 in beta-tubulin isotype 1. Mol Biochem Parasitol. 1994;63:299-303.

39. Mottier M, Prichard RK. Genetic analysis of a relationship between macrocyclic lactone and benzimidazole anthelmintic selection on Haemonchus contortus. Pharmacogenet Genomics. 2008;18:12940.

40. Silvestre A, Cabaret J. Mutation in position 167 of isotype 1 betatubulin gene of Trichostrongylid nematodes: role in benzimidazole resistance? Mol Biochem Parasitol. 2002;120:297-300.

41. Bennett AB, Anderson TJC, Barker GC, Michael E, Bundy DAP. Sequence variation in the Trichuris trichiura $\beta$-tubulin locus: implications for the development of benzimidazole resistance. Int $\mathbf{J}$ Parasitol. 2002;32:1519-28.

42. Diawara A, Drake LJ, Suswillo RR, Kihara J, Bundy DAP, Scott ME, et al. Assays to detect beta-tubulin codon 200 polymorphism in Trichuris trichiura and Ascaris lumbricoides. PLoS Negl Trop Dis. 2009;3:e397.

43. Diawara A, Schwenkenbecher JM, Kaplan RM, Prichard RK. Molecular and biological diagnostic tests for monitoring benzimidazole resistance in human soil-transmitted helminths. Am J Trop Med Hyg. 2013;88:1052-61.

44. Diawara A, Halpenny CM, Churcher TS, Mwandawiro C, Kihara J, Kaplan RM, et al. Association between response to albendazole treatment and $\beta$-tubulin genotype frequencies in soil-transmitted helminths. PLoS Negl Trop Dis. 2013;7:e2247. An increase in the frequency of alleles associated with resistance was observed in T. trichiura isolates following albendazole treatment.

45. Hansen TV, Thamsborg SM, Olsen A, Prichard RK, Nejsum P. Genetic variations in the beta-tubulin gene and the internal transcribed spacer 2 region of Trichuris species from man and baboons. Parasit Vectors. 2013;6:236.

46. Mottier L, Alvarez L, Ceballos L, Lanusse C. Drug transport mechanisms in helminth parasites: passive diffusion of benzimidazole anthelmintics. Exp Parasitol. 2006;113:49-57.

47. Geary T, Blair K, Ho N, Sims S, Thompson D. Biological functions of nematode surfaces. In: Boothroyd J, Komuniecki R, editors. Mol. approaches to Parasitol. New York: Wiley-Liss; 1995. p. 5776.

48. Helbæk H. Tollund mandens sidste måltid. Aarbøger Nord. Oldkynd. og Hist. Kongelige Nordiske Oldskriftsselskab; 1950. p. 311-41.

49. Rácz SE, De Aráujo EP, Jensen E, Mostek C, Morrow JJ, Van Hove ML, et al. Parasitology in an archaeological context: analysis of medieval burials in Nivelles Belgium. J Archaeol Sci. 2014;53: 304-15.

50. Nansen P, Jørgensen RJ. Parasite eggs identified in material from archaeological excavations in Ribe (the Viking age). Nord Vet Med. 1977;29:263-6.

51. Mitchell PD. Human parasites in medieval Europe: lifestyle, sanitation and medical treatment. Adv Parasitol. 2015;90:1-32.

52. Goncalves MLC, Araujo A, Ferreira LF. Human intestinal parasites in the past: new findings and a review. Mem Inst Oswaldo Cruz. 2003;98:103-18.

53. Loreille O, Roumat E, Verneau O, Bouchet F, Hänni C. Ancient DNA from Ascaris: extraction amplification and sequences from eggs collected in coprolites. Int J Parasitol. 2001;31:1101-6.

54. Oh CS, Seo M, Chai JY, Lee SJ, Kim MJ, Park JB, et al. Amplification and sequencing of Trichuris trichiura ancient DNA extracted from archaeological sediments. J Archaeol Sci. 2010;37: 1269-73.

55. Søe MJ, Nejsum P, Fredensborg BL, Kapel CMO. DNA typing of ancient parasite eggs from environmental samples identifies human and animal worm infections in Viking-age settlement. J Parasitol. 2015;101:57-63. Identification of T. trichiura infections in $\mathbf{1 0 0 0}$ year old contexts by analysis of egg morphology and DNA sequence. Protocols for DNA extraction, PCR using overlapping primers and sequencing are described.

56. Lv Z, Wu Z, Zhang L, Ji P, Cai Y, Luo S, et al. Genome mining offers a new starting point for parasitology research. Parasitol Res. 2015;114:399-409.

$57 . \bullet$ Foth BJ, Tsai IJ, Reid AJ, Bancroft AJ, Nichol S, Tracey A, et al. Whipworm genome and dual-species transcriptome analyses provide molecular insights into an intimate host-parasite interaction. Nat Genet. 2014;46:693-700. Publication describing the genome of $T$. trichiura and the genome and transcriptome of $T$. muris at various life stages and in particular parasite body parts. Immunomodulator mechanisms are identified along with new potential drug targets against trichuriasis.

$58 . \bullet$ Jex AR, Nejsum P, Schwarz EM, Hu L, Young ND, Hall RS, et al. Genome and transcriptome of the porcine whipworm Trichuris suis. Nat Genet. 2014;46:701-6. Publication describing the male and female genome $T$. suis and the transcriptome of the two sexes, body parts and different life stages. The protein-encoding gene set is presented along with molecules of importance for host- 
parasite interaction as well as the role of small noncoding RNAs in gene regulation.

59. Agüero F, Al-Lazikani B, Aslett M, Berriman M, Buckner FS, Campbell RK, et al. Genomic-scale prioritization of drug targets: the TDR Targets database. Nat Rev Drug Discov. 2008;7:900-7.

60. Britton C, Winter AD, Gillan V, Devaney E. MicroRNAs of parasitic helminths - identification, characterization and potential as drug targets. Int J Parasitol Drugs Drug Resist. 2014;4:85-94.

61. Allentoft ME, Sikora M, Sjögren K-G, Rasmussen S, Rasmussen $\mathrm{M}$, Stenderup J, et al. Population genomics of Bronze Age Eurasia. Nature. 2015;522:167-72.

62. Merker M, Blin C, Mona S, Duforet-Frebourg N, Lecher S, Willery E, et al. Evolutionary history and global spread of the Mycobacterium tuberculosis Beijing lineage. Nat Genet. 2015;47: 242-9.

63. Dey SS, Kester L, Spanjaard B, Van A. Integrated genome and transcriptome sequencing from the same cell. Nat Biotechnol. 2014;33:285-9.

64. Sandberg R. Entering the era of single-cell transcriptomics in biology and medicine. Nat Methods. 2014;11:22-4.

65. Carlsgart J, Roepstorff A, Nejsum P. Mulitplex PCR on single unembryonated Ascaris (roundworm) eggs. Parasitol Res. 2009;104:939-43.

66. Holroyd N. Sanchez-Flores a. Producing parasitic helminth reference and draft genomes at the Wellcome Trust Sanger Institute. Parasite Immunol. 2012;34:100-7.

67. Vejzagić N, Thamsborg SM, Kringel H, Roepstorff A, Bruun JM, Kapel CMO. In vitro hatching of Trichuris suis eggs. Parasitol Res. 2015;114:2705-14.

68. Koyama K. Evidence for bacteria-independent hatching of Trichuris muris eggs. Parasitol Res. 2013;112:1537-42.

69. Hayes KS, Bancroft AJ, Goldrick M, Portsmouth C, Roberts IS, Grencis RK. Exploitation of the intestinal microflora by the parasitic nematode Trichuris muris. Science. 2010;328:1391-4.

70. Marcilla A, Trelis M, Cortés A, Sotillo J, Cantalapiedra F, Minguez $\mathrm{M}$, et al. Extracellular vesicles from parasitic helminth contain specific excretory/secretory proteins and are internalized in intestinal host cells. PLoS One. 2012;7:e45974.

71. Twu O, de Miguel N, Lustig G, Stevens G, Vashisht A, Wohlschlegel J, et al. Trichomonas vaginalis exosomes deliver cargo to host cells and mediate host:parasite interactions. PLoS Pathog. 2013;9:e1003482.
72. Bernal D, Trelis M, Montaner S, Cantalapiedra F, Galiano A, Hackenberg M, et al. Surface analysis of Dicrocoelium dendriticum. The molecular characterization of exosomes reveals the presence of miRNAs. J Proteome. 2014;105:232-41.

73. Buck A, Coakley G, Simbari F, McSorley H, Quintana J, Le Bihan $\mathrm{T}$, et al. Exosomes secreted by nematode parasites transfer small RNAs to mammalian cells and modulate innate immunity. Nat Commun. 2014;5:5488.

74. Hansen TVA. H K, AR W, Nejsum P. Secretion of RNA-containing extracellular vesicles by the porcine whipworm, Trichuris suis. J Parasitol. 2015;101:336-40.

75. Araujo A, Reinhard KJ, Ferreira LF, Gardner SL. Parasites as probes for prehistoric human migrations? Trends Parasitol. 2008;24:112-5.

76. Rasmussen M, Anzick SL, Waters MR, Skoglund P, DeGiorgio M, Stafford TW, et al. The genome of a Late Pleistocene human from a Clovis burial site in western Montana. Nature. 2014;506:225-9.

77. Raghavan M, DeGiorgio M, Albrechtsen A, Moltke I, Skoglund P, Korneliussen TS, et al. The genetic prehistory of the New World Arctic. Science. 2014;345:1255832-32.

78. Notomi T, Okayama H, Masubuchi H, Yonekawa T, Watanabe K, Amino N, et al. Loop-mediated isothermal amplification of DNA. Nucleic Acids Res. 2000;28:E63.

79. Søe M, Rohde M, Mikkelsen J, Warthoe P. IsoPCR: an analytically sensitive, nested, multiplex nucleic acid amplification method. Clin Chem. 2013;439:3-6.

80. Søe MJ, Warthoe P. RT-isoPCR: nested, high multiplex mRNA amplification. Analyst. 2013;138:5871-4.

81. Watts MR, James G, Sultana Y, Ginn AN, Outhred AC, Kong F, et al. A loop-mediated isothermal amplification (LAMP) assay for Strongyloides stercoralis in stool that uses a visual detection method with SYTO-82 fluorescent dye. Am J Trop Med Hyg. 2014;90: 306-11.

82. Le TH, Nguyen NTB, Truong NH, Van De N. Development of mitochondrial loop-mediated isothermal amplification for detection of the small liver fluke Opisthorchis viverrini (opisthorchiidae; trematoda; platyhelminthes). J Clin Microbiol. 2012;50:1178-84.

83. Park S, Zhang Y, Lin S, Wang TH, Yang S. Advances in microfluidic PCR for point-of-care infectious disease diagnostics. Biotechnol Adv. 2011;29:830-9.

84. Rand KH, Tremblay EE, Hoidal M, Fisher LB, Grau KR, Karst SM. Multiplex gastrointestinal pathogen panels: implications for infection control. Diagn Microbiol Infect Dis. 2015;82:154-7. 